\title{
REMPI spectroscopy of cytosine
}

\author{
E. Nir, M. Müller, L.I. Grace, M.S. de Vries * \\ Department of Chemistry and Biochemistry, University of California at Santa Barbara, Santa Barbara, CA 93106, USA
}

Received 7 January 2002

\begin{abstract}
We report resonant two-photon ionization spectra of laser desorbed, jet cooled, cytosine, 1-methyl cytosine, 5 -methyl cytosine, and dimers of these. Unlike other pyrimidine bases, cytosine exhibits vibronic spectra with sharp features in two spectral regions, separated by about $5000 \mathrm{~cm}^{-1}$. We interpret these as being due to two tautomeric forms, one keto and one enol. The dimers absorb at wavelengths that are intermediate between those of the two monomer forms. By UV-UV hole burning we determined the numbers of isomers contributing to each spectrum and by delayed two color ionization we determined triplet lifetimes. We observed hydrogen transfer between bases both in collisions between monomers and after photo-excitation in clusters. (c) 2002 Published by Elsevier Science B.V.
\end{abstract}

\section{Introduction}

Studying biomolecular building blocks in the gas phase makes it possible to distinguish their intrinsic molecular properties from those imposed by the biological environment. This concept is particularly intriguing for the DNA bases because it allows us to study their hydrogen bonding interactions in individual base pairs separate from the macro molecular backbone and solvent effects. Brady et al. [1] studied the vibronic spectra of the pyrimidine bases uracil and thymine in the gas phase and found them to be structureless with a broad absorption onset. They interpreted this observation as being due to interactions between multiple excited states. More recently we have used

\footnotetext{
${ }^{*}$ Corresponding author. Fax: +805-893-4120.

E-mail address: devries@chem.ucsb.edu (M.S. de Vries).
}

laser desorption jet-cooling to obtain the vibronic spectra of the purine bases, which we found to contain sharp, well-resolved features. We have reported REMPI spectra of guanine [2] and several of its derivatives as well as guanosine [3] and adenine [4]. Additional data on the purine bases have been reported by other authors [5-7]. We have used the guanine chromophore to study GG and GC base pairs as clusters in the supersonic beam [8]. We have used hole burning spectroscopy to distinguish and identify different tautomers and different cluster structures [9]. Such detailed spectroscopy of the purine bases provides new insights into DNA photochemistry and base pairing dynamics. However, significant further details could be revealed if similarly detailed spectroscopy of some or all of the pyrimidine bases was accessible as well. We are now reporting the first REMPI spectra of laser desorbed cytosine and several of its derivatives exhibiting sharp features. We observed 
sharp spectra in two different spectral regions which we interpret as being due to different tautomers of cytosine. We also report spectra of cytosine dimers, representing $\mathrm{CC}$ base pairs, and evidence for hydrogen transfer between individual cytosine bases.

\section{Experimental}

The experimental setup has been described in detail elsewhere [10]. In brief, material is laser desorbed from a graphite sample probe in front of a pulsed nozzle. Typical laser fluences are of the order of $1 \mathrm{~mJ} / \mathrm{cm}^{2}$ or less, which is significantly less than the fluences normally used for ablation. Downstream, ionization lasers intersect the beam inside the source region of a reflectron time of flight (TOF) mass spectrometer. Light from two $\mathrm{Nd}$ :YAG-pumped dye lasers, as well as from an excimer laser, used for two-photon ionization and for spectral hole burning, intersects the beam at right angles. By monitoring specific mass peaks while varying the two-photon ionization wavelength, we obtain mass selected excitation spectra. We achieve spectral hole burning by implementing a delay of 200-600 ns between the two dye laser pulses. This results in two peaks in the TOF spectrum, which we can monitor individually. The first laser pulse then serves as a 'burn' laser, while the second serves as a 'probe' laser. When both lasers are tuned to a resonance of the same isomer population the burn laser causes a decrease in the signal of the probe laser.

\section{Results}

Figs. 1 and 2 show the REMPI spectra of cytosine (C), 1-methyl cytosine (1mC) and 5-methylcytosine $(5 \mathrm{mC})$. For $\mathrm{C}$ and $5 \mathrm{mC}$ we recorded sharp features in two distinct spectral regions: Region A from 31000 to $32500 \mathrm{~cm}^{-1}$, shown in Fig. 1, and region B from 36000 to $37500 \mathrm{~cm}^{-1}$, shown in Fig. 2. For $1 \mathrm{mC}$ we found features only in region $\mathrm{A}$. We recorded the spectra by two color ionization, with the first photon from a dye laser and the second photon from an ArF excimer laser at $193 \mathrm{~nm}$. The spectra in region $\mathrm{B}$ could also be recorded with one color two-photon ionization while the spectra in region A could not. This suggests that the ionization potential for the vari-

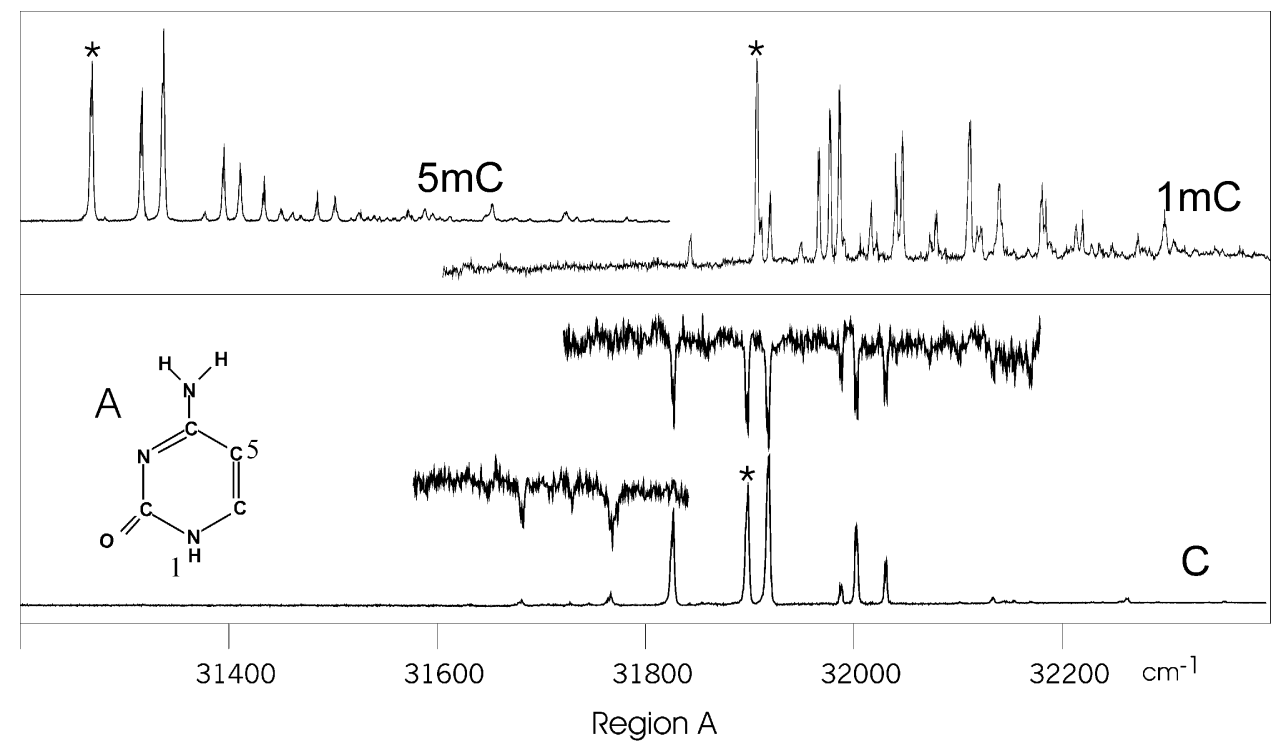

Fig. 1. REMPI spectra in frequency region $\mathrm{A}$ of cytosine $(\mathrm{C}), 1$-methylcytosine $(1 \mathrm{mC})$ and 5-methylcytosine (5mC). Also shown are the hole burning spectra, revealing two isomers for cytosine. Asterisks indicate peaks that were used for lifetime measurements. 


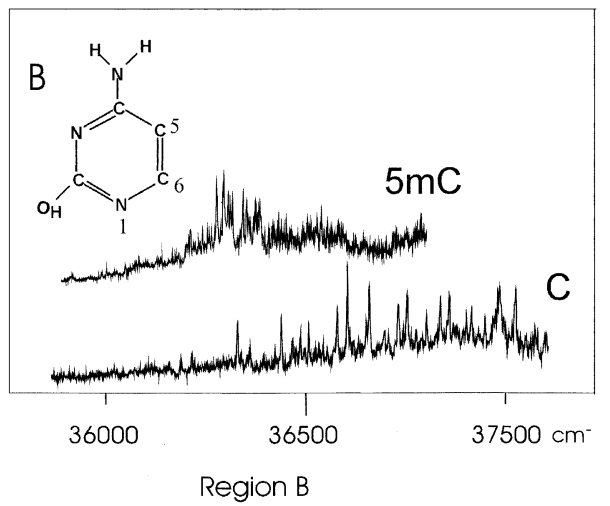

Fig. 2. REMPI spectra in frequency region $B$ of cytosine (C) and 5-methylcytosine $(5 \mathrm{mC})$.

ous cytosine compounds is between 8 and $9 \mathrm{eV}$, representing twice the photon energy in regions $\mathrm{A}$ and $\mathrm{B}$, respectively. We carefully scanned the entire spectral range between regions $\mathrm{A}$ and $\mathrm{B}$, employing two color ionization, without observing any ions.

Although the lifetime of photo-excited cytosine in solution is of the order of 1 ps [11], the possibility for intersystem crossing to a long-lived triplet state exists in the gas phase. We observe the transition to the triplet state by first exciting the molecules to the singlet state, and subsequently ionizing them with a second laser after a variable delay. By recording the ion signal as a function of the delay we can measure the lifetime of the triplet state. We have done this for the spectra in region A. For these spectra there is no ion signal from the excitation laser alone. As a second photon we used $193 \mathrm{~nm}$ light from an excimer laser. The ionizing laser alone, at the fluences used, produces only a very small ion signal, which can be subtracted as background. Fig. 3 shows the signal as a function of delay time, with the dye laser tuned to the 31898,31907 and $31268 \mathrm{~cm}^{-1}$ resonance, respectively for $\mathrm{C}, 1 \mathrm{mC}$ and $5 \mathrm{mC}$, as marked with asterisks in Fig. 1. The curves can be fit to a single exponential decay with lifetimes of 290, 380 and 1095 ns, respectively. The contribution to the decay signal from the fact that excited molecules leave the interaction region can be neglected because we used an ionizing laser spot of more

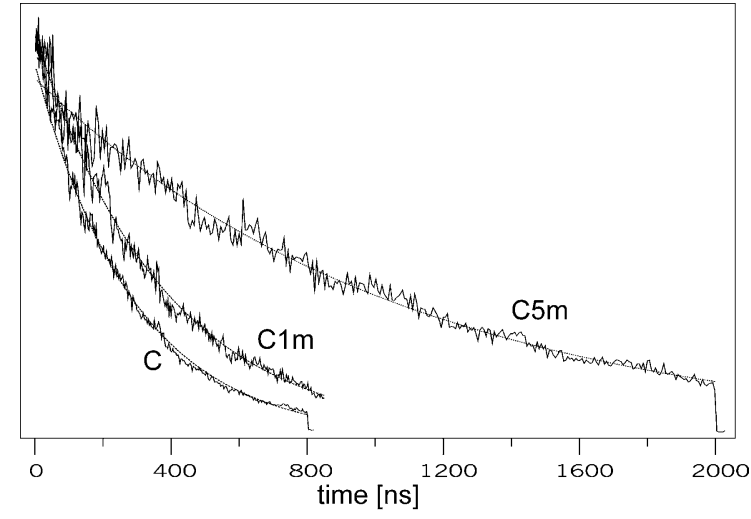

Fig. 3. Signal as a function of delay time between excitation laser and ionization laser for $\mathrm{C}, 1 \mathrm{mC}$, and $5 \mathrm{mC}$. Dotted lines are single exponential least squares fits.

than $5 \mathrm{~mm}$ along the direction of the molecular beam. Under these conditions it takes an average molecule more than $8 \mu$ s to cross the laser beam.

The spectrum of cytosine in region A shows a number of peaks at lower energy with an intensity a factor of 30 less than those at higher energy. UV-UV hole burning shows this series of peaks to belong to a different ground state population, as shown in Fig. 1. This could be due to a different isomeric form, hot bands or dissociating clusters with the drive gas. The latter can be excluded because we recorded an identical spectrum when we replaced $\mathrm{Ar}$ by $\mathrm{CO}_{2}$ as the drive gas. Because the excitation in this region is to a state at less than half the ionization energy, a second more energetic photon is required to ionize the excited molecules. In the hole burning experiments we used $193 \mathrm{~nm}$ light from an excimer laser with a delay to ionize excited molecules that had crossed to the triplet state. The delay between the analysis laser and the ionizing laser $(193 \mathrm{~nm})$ was kept at $20 \mathrm{~ns}$ in order to ionize the largest possible triplet population created by the analysis laser. In order to prevent ionization of molecules that had occupied the triplet state after excitation by the burn laser, we applied a delay between burn and analysis laser long enough to allow decay of most of that triplet state population. The delays employed for $\mathrm{C}$, $1 \mathrm{mC}$, and $5 \mathrm{mC}$ were 1700,1700 , and $4000 \mathrm{~ns}$, respectively. 


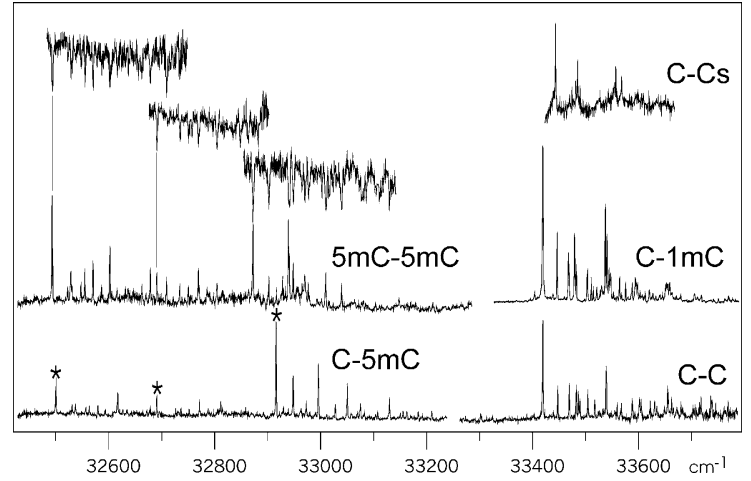

Fig. 4. REMPI spectra of cytosine clusters. Hole burning spectra show different isomers for the $5 \mathrm{mC}-5 \mathrm{mC}$ cluster. Asterisks indicate the origins of different $\mathrm{C}-5 \mathrm{mC}$ clusters, as found by hole burning. $\mathrm{C}-\mathrm{Cs}$ indicates the cytosine-cytidine cluster.

Fig. 4 shows REMPI spectra recorded at the masses of dimers and mixed dimers. Here we expect low frequency vibrations due to hydrogen bond vibrations, and the red-most peak may be the origin. A number of dimer structures are possible [12-14]. We have performed spectral hole burning experiments to determine the number of structures in these spectra. We found that the $\mathrm{C}-\mathrm{C}$ and $\mathrm{C}-1 \mathrm{mC}$ spectra each are due to a single isomer, while the $\mathrm{C}-5 \mathrm{mC}$ and $5 \mathrm{mC}-5 \mathrm{mC}$ spectra each consist of contributions from three isomers. An example of the hole burning spectra appears in Fig. 4 for the $5 \mathrm{mC}-5 \mathrm{mC}$ cluster. The figure also shows a spectrum of a cluster of cytosine with the nucleoside, cytidine.

\section{Discussion}

An interpretation of these spectra is that those in region $\mathrm{A}$ are due to the cytosine moiety in the keto tautomeric form while those in region $\mathrm{B}$ are due to the enol form. These structures are shown as structures A and B, respectively, in Figs. 1 and 2. This interpretation is supported by the following three observations. First, in $1 \mathrm{mC}$ the enol form is tautomerically blocked and the molecule does not absorb in region B. In $5 \mathrm{mC}$ both tautomers are still possible to occur and we observe spectra in both regions. Sambrano et al. [15] predict that the amino-oxo and amino-hydroxy forms are the two most stable tautomers in the gas phase, with a $0.65 \mathrm{kcal} \mathrm{mol}^{-1}$ energy difference. The second argument follows from comparison with the spectra of pyridone (2PY) and 2hydroxypyridine (2HP). These compounds can be viewed as models for keto and enol cytosine, respectively, and their $\mathrm{S}_{1}$ origins were found at 29935 and $36316 \mathrm{~cm}^{-1}$, respectively [16-19]. An even closer comparison would be with analogous forms of pyrimidine, but no vibronic spectra of these have yet been reported. Although 2PY and 2HP contain just one nitrogen in the ring, rather than two, their spectra show that a $6500 \mathrm{~cm}^{-1}$ difference in absorption between the two tautomers is possible, even though the enol-keto tautomer splitting in the purine DNA bases is more than an order of magnitude smaller. In the third place we note that the two proposed structures are the ones that should be expected to predominate in our beam. Brown et al. [20] identified these tautomers as predominant species in equal abundances in a supersonic expansion by means of microwave spectroscopy. A third form in which a hydrogen from $\mathrm{NH}_{2}$ has migrated to the $\mathrm{N} 3$ position was found with only minimal population. Structures A and B were also predicted to be most stable in the gas phase in ab initio calculations [21], even though structure A predominates in the solid and in solution.

It is not clear whether we observe the $0-0$ transition in all of the monomer spectra. We would expect the lowest in-plane vibrational frequencies to be of the order of a few hundred wave numbers. By comparison with matrix isolation spectra and calculations, the first in-plane vibration in the ground state would be expected at about $340 \mathrm{~cm}^{-1}$ [22-24]. We have performed calculations at the MP2/6G 311 level, obtaining similar results. However, the experimentally observed peaks are much closer together. Furthermore the red-most peak in each spectrum is not the largest one. A large geometry change in the excited state, which could account for these observations, is unlikely for an aromatic ring molecule. Another possible explanation involves interactions between multiple excited states. This has also been proposed to explain the spectrum of adenine, which is also characterized by a narrow range with low 
frequency spacings [4]. Assuming that the observed transition is of $\pi \pi^{*}$ character, the existence of curve crossings has been proposed with an $n \pi^{*}$ state or with a $\pi \sigma^{*}$ state or both $[25,26]$. Such curve crossings, or conic intersections, could lead to predissociation, explaining the narrow range of the spectra. They could also lead to broad adiabatic potential energy surfaces, causing significantly lower frequency vibrations. These interactions are thought to be important in providing pathways to internal conversion, shortening the excited state lifetime and protecting the DNA bases from photochemistry. Similar interactions may be underlying the broad spectra in the other two pyrimidine bases, $U$ and T. Guanine appears to be exceptional in this respect, in that it exhibits a lower $\pi \pi^{*}$ state. The more highly congested nature of the spectra in region $\mathrm{B}$ could be due to the fact that at higher energy there are more opportunities for interaction with other excited states. An additional complication might be that, if these are indeed spectra of the enol form, we could expect at least two possible conformations of the $\mathrm{OH}$ group leading to overlapping spectra.

The similarity between the $\mathrm{C}-\mathrm{C}$ and the $\mathrm{C}-1 \mathrm{mC}$ spectra suggests a common cluster structure. This would imply that the $\mathrm{C}-\mathrm{C}$ structure cannot involve hydrogen bonding at the N1 position. The fact that methyl substitution in the $\mathrm{C} 5$ position increases the number of observed structures is intriguing since methyl substitution limits the possibilities for hydrogen bonding rather than increasing them. On the other hand, Sponer et al. [14] point out that methylation may increase the number of stacking interactions. All dimer spectra appear at frequencies between those of regions A and $\mathrm{B}$. This is remarkable, since in other base pairs, i.e., GG and GC, we have not observed such large shifts with respect to the monomer spectra [8]. For example, we observed two $\mathrm{G}-\mathrm{G}$ origins at 33103 and $33282 \mathrm{~cm}^{-1}$, while we found origins of different $G$ tautomers at 32868, 33272 and $33912 \mathrm{~cm}^{-1}$ [9] The large shift in the cytosine dimers leads one to speculate that the cytosine clusters might have a mixed tautomeric character. This could be the result of a hydrogen bonding configuration in which the hydrogen atom is shared between a nitrogen atom on one monomer
- corresponding to a keto structure - and an oxygen atom on the other monomer - corresponding to an enol structure. To address this issue we will carry out IR-UV hole burning experiments on the clusters as well. Such measurements can also help elucidate the cluster structures themselves.

We observed evidence for proton transfer between all cytosine bases at high rates both with and without photo-excitation. We observe proton transfer with photo-excitation by exciting and ionizing cytosine clusters at their resonant wavelengths, and measuring the signal of a fragment at the mass of a monomer plus one mass unit, as shown in Fig. 5. The resonant wavelength establishes that the first photon is absorbed by the cytosine cluster, and at the same time the observed product channel establishes that a proton transfer has taken place in the process. We have also observed hydrogen exchange between neutral cytosine monomers without photo-excitation. This observation follows from experiments with mixtures of cytosine and methyl cytosine in which one of the two compounds was deuterated. Following laser desorption and jet-cooling we could observe deuterated molecules for both compounds. In principle this exchange could take place on the

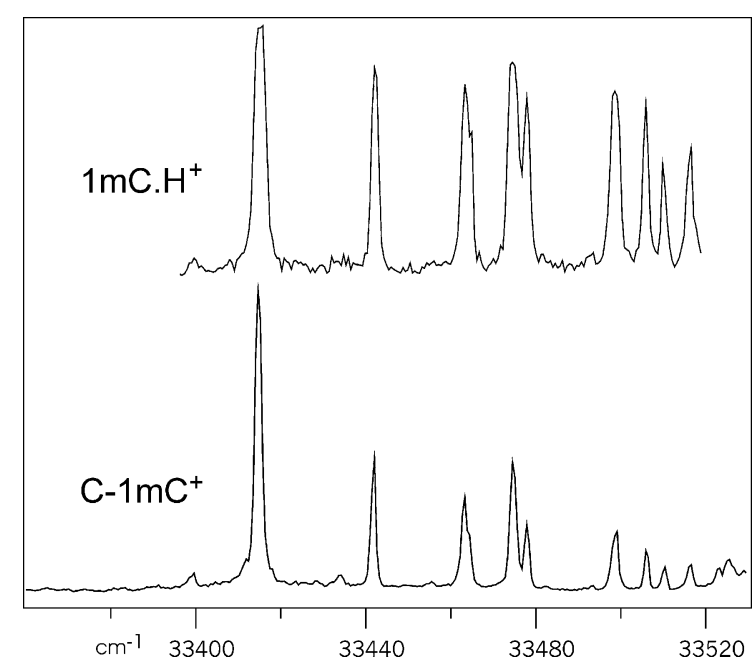

Fig. 5. REMPI spectra of the cytosine-1-methylcytosine cluster, recorded at the parent mass (bottom trace) and $t$ the mass of fragment with mass of cytosine +1 (top trace). The top spectrum is saturated because it was recorded at higher laser fluence. 
sample, however, these samples were prepared without any solvent as loose mixtures of separate crystalline powders. Therefore, it seems more likely that the exchange takes place in the desorption step or in the multiple collisions occurring in the high-pressure region of the supersonic expansion.

\section{Summary}

Cytosine, and several of its methyl derivatives exhibit vibronic spectra with sharp features in two spectral regions, separated by about $5000 \mathrm{~cm}^{-1}$. We interpret those as being due to two tautomeric forms, one keto and one enol. Dimers of these compounds absorb at intermediate wavelengths, pointing to a structure with possibly mixed tautomeric character. We are undertaking IR-UV hole burning experiments to verify the tautomer assignments and to elucidate the cluster structures. This work has been supported by the Israel Science Foundation, founded by The Israel Academy of Sciences and Humanities.

\section{References}

[1] B.B. Brady, L.A. Peteanu, D.H. Levy, Chem. Phys. Lett. 147 (1988) 538.

[2] E. Nir, L.I. Grace, B. Brauer, M.S. de Vries, J. Am. Chem. Soc. 121 (1999) 4896.

[3] E. Nir, K. Kleinermanns, L.I. Grace, M.S. de Vries, J. Am. Chem. Soc. 122 (2000) 8091.

[4] E. Nir, L.I. Grace, M.S. de Vries, J. Phys. Chem. A 105 (2001) 5106.
[5] D.C. Luhrs, J. Viallon, I. Fischer, Phys. Chem. Chem. Phys. 3 (2001) 1827.

[6] N.J. Kim, G. Jeong, Y.S. Kim, J. Sung, S.K. Kim, Y.D. Park, J. Chem. Phys. 113 (2000) 10051.

[7] F. Piuzzi, M. Mons, I. Dimicoli, B. Tardivel, Q. Zhao, Chem. Phys. 270 (2001) 205.

[8] E. Nir, K. Kleinermanns, M.S. de Vries, Nature 408 (2000) 949.

[9] E. Nir, C. Janzen, P. Imhof, K. Kleinermanns, M.S. de Vries, J. Chem. Phys. 115 (2001) 4604.

[10] G. Meijer, M.S. de Vries, H.E. Hunziker, H.R. Wendt, Appl. Phys. B 51 (1990) 395.

[11] A. Reuther, H. Iglev, R. Laenen, A. Laubereau, Chem. Phys. Lett. 325 (2000) 360.

[12] M. Elstner, P. Hobza, T. Frauenheim, S. Suhai, E. Kaxiras, J. Chem. Phys. 114 (2001) 5149.

[13] M. Kabelá, P. Hobza, J. Phys. Chem. B 105 (2001) 5804.

[14] J. Sponer, J. Leszczynski, P. Hobza, Theochem 573 (2001) 43.

[15] J.R. Sambrano, A.R. de Souza, J.J. Queralt, M. Oliva, J. Andres, Chem. Phys. 264 (2001) 333.

[16] G.M. Florio, C.J. Gruenloh, R.C. Quimpo, T.S. Zwier, J. Chem. Phys. 113 (2000) 11143.

[17] A. Held, B.B. Champagne, D.W. Pratt, J. Chem. Phys. 95 (1991) 8732.

[18] A. Held, D.W. Pratt, J. Chem. Phys. 96 (1992) 4869.

[19] A. Held, D.W. Pratt, J. Am. Chem. Soc. 115 (1993) 9708.

[20] R.D. Brown, P.D. Godfrey, D. McNaughton, A. Pierlot, J. Am. Chem. Soc. 111 (1989) 2308.

[21] S. Morpurgo, M. Bossa, G.O. Morpurgo, Chem. Phys. Lett. 280 (1997) 233.

[22] I.R. Gould, M.A. Vincent, I.H. Hillier, L. Lapinski, M. Nowak, Spectrochim. Acta, Part A (Molecular Spectroscopy) 48A (1992) 811.

[23] I.R. Gould, N.A. Burton, R.J. Hall, I.H. Hillier, Theochem 331 (1995) 147.

[24] R. Santamaria, E. Charro, A. Zacarias, M. Castro, J. Comput. Chem. 20 (1999) 511.

[25] A. Broo, J. Phys. Chem. A 102 (1998) 526.

[26] A.L. Sobolewski, W. Domcke, Chem. Phys. Lett. 315 (1999) 293. 